\title{
Platelet Count Applicability in Predicting the Degree of ST-Segment Depression and Coronary Flow Rate in Acute Myocardial Infarction Patients
}

\author{
Roghaiyeh Afsargharehbagh $^{1^{*} \text {, }}$, Kamal Khademvatani ${ }^{2}$, Tohid Yahyapoor ${ }^{2}$, Aliakbar Nasiri ${ }^{3}$, Mahmood \\ Moosazadeh ${ }^{4}$, Motahareh Kheradmand ${ }^{5}$, Mahdi Afshari ${ }^{6}$, Mir Hossein Seyed-Mohammadzad ${ }^{*}$ \\ 'Department of Interventional Cardiology, Shohada Hospital, Urmia University of Medical Sciences, Urmia, Iran \\ ${ }^{2}$ School of Medicine, Urmia University of Medical Sciences, Urmia, Iran \\ ${ }^{3}$ Department of Anesthesiology, Urmia University of Medical Sciences, Urmia, Iran \\ ${ }^{4}$ Health Sciences Research Centre, Addiction Institute, Mazandaran University of Medical Science, Sari, Iran \\ ${ }^{5}$ Health Sciences Research Center, School of Health, Mazandaran University of Medical Sciences, Sari, Iran \\ ${ }^{6}$ Department of Community Medicine, Zabol University of Medical Sciences, Zabol, Iran
}

*Correspondence to

Roghaiyeh Afsargharehbagh,

Tel: +989143029126

Email: r.afsargarebag@gmail.com;

MirHossein Seyed-Mohammadzad,

Email: mohammadzad.h@umsu.ac.ir

Received November 11, 2019 Accepted November 25, 2020

Published online 30 Septemeber 2020

\begin{abstract}
Introduction: It is still unclear whether platelet count can predict the outcomes of acute myocardial infarction. In this study, we assessed the relationship between the initial platelet count on the degree of ST-segment depression and coronary flow rate among patients with $\mathrm{MI}$ who underwent percutaneous coronary intervention $(\mathrm{PCI})$.

Methods: In this study, a total of 218 patients suffering from $\mathrm{MI}$, who underwent primary $\mathrm{PCl}$ during 2016-2017 (Seyed-Shohada hospital, Urmia, Iran) were selected by consensus method. Demographic information and past medical history such as diabetes mellitus (DM), cigarette smoking, using Integrilin, and door-to-balloon (DTB) time were recorded. All patients were investigated in terms of cell blood count. Serial electrocardiogram (ECG) was also performed and the degree of ST-segment elevation was measured.

Results: The mean (SD) age of participants was 58.67 (11.44) years. The initial platelet count was similar between patients with and without improvement in the ST-segment $(P=0.275)$. There was no significant difference regarding thrombolysis in myocardial infarction (TIMI) between patients with and without improved ST-segment $(P=0.380)$.

Conclusion: According to our results, the initial platelet count in patients who underwent angioplasty was not associated with coronary flow and echocardiographic responses to treatment.

Keywords: Platelet count, ST-segment depression, Coronary flow, Acute myocardial infarction
\end{abstract}

\section{Introduction}

Cardiovascular diseases (CVDs) are the most common causes of death worldwide. ${ }^{1}$ Despite the progresses in diagnosis and treatment, CVDs are still main health problems in developed and developing countries. Prevalence of myocardial infarction (MI) has been increased in Iran during the last decade. ${ }^{2}$ During MI, unstable vascular plaques rupture occurs leading to platelet reactions and different degrees of thrombosis, which is responsible for clinical complications in short time. ${ }^{3}$ Anti-platelet therapies have had clinical benefits in patients with MI. The initial measurement of platelet count during the first stages of the MI has been suggested as a suitable criterion for predicting the response to fibrinolytic agents. ${ }^{4,5}$

Coronary angiography which is the standard diagnostic method for coronary disorders helps to select the best choices of pharmaceutical and surgical treatments, such as percutaneous coronary intervention (PCI) and bypass surgery. ${ }^{6}$ These interventional procedures are applied in combination with anti-platelet and anti-coagulant therapy in patients with and without ST-segment elevation to prevent thrombosis in the site of intervention. ${ }^{7}$ The association between platelet count and acute MI outcomes has been controversial. ${ }^{89}$ The thrombolysis in myocardial infarction (TIMI) which is

(c) 2020 The Author(s); Published by Zabol University of Medical Sciences. This is an open-access article distributed under the terms of the Creative Commons Attribution License (http://creativecommons.org/licenses/by/4.0), which permits unrestricted use, distribution, and reproduction in any medium, provided the original work is properly cited. 
usually used for assessment of the degree of coronary flow indicates the speed and amount of the contrast material transmitted through coronary vessels. ${ }^{10}$ Accordingly, this study was carried out to investigate whether the initial platelet count can be a predictor for improvements of the ST-segment and TIMI after PCI.

\section{Materials and Methods \\ Participants}

Patients with acute MI, who underwent PCI during 2016-2017 in Seyed-Shohada hospital, Urmia, Iran, were entered into the study by consensus method. Patients received thrombolytics and those without indication for stent replacement (based on angiographic results) were excluded from the study. After providing an informed consent, a pre-designed checklist including such information as gender, age, history of diabetes mellitus (DM), cigarette smoking, use of Integrilin, and door-toballoon (DTB) time was completed. Then, a blood sample was collected from each patient and complete blood cell count was measured. 12-lead electrocardiogram (ECG) was conducted and type of MI, as well as the degree of STsegment elevation were measured. After confirming by a team of interventional cardiologists, patients underwent primary PCI. Platelet count and the degree of ST-segment depression during the treatment were recorded from the hospital files. In addition, the angiographic results of all patients were reviewed by another interventional cardiologist, who was blinded to the baseline information of the patients to determine the degree of TIMI.

\section{Statistical Methods}

Data were described by mean (standard deviation). Categorical and continuous variables were compared between the study groups using chi-square test or $t$ test, respectively. The association between platelet count and the investigated outcomes (ST-depression and TIMI) was determined using logistic regression models controlling the effect of potential confounders. STATA software version 14.1 was used for statistical analysis. P-value less than 0.05 was considered statistically significant.

\section{Results}

Totally, 218 patients (age range: 33-88 years) were investigated in this study. The mean (SD) age of participants was 58.67 (11.44) years, and 182 (83.49\%) subjects were male. During the treatment, 198 (90.83\%) patients experienced more than $50 \%$ depression in STsegment, while $20(9.18 \%)$ remained with less than $50 \%$ ST-segment depression. There was a significant difference in gender distribution between patients with or without $>50 \%$ ST-depression, and females showed more pronounced depression rate (Table $1,18.18 \%$ vs 0 , $P=0.051)$. A higher desirable DTB time was observed in patients with $>50 \%$ ST-depression ( 88.89 vs $65, P=0.008$ ). Regarding other demographic and clinical factors, no difference was observed between the two groups.

As illustrated in Table 2, no statistically significant difference was observed between patients with and without desired ST-segment depression in terms of platelet count $(P=0.275)$. The crude and adjusted (controlling for gender, DTB time, and type of MI) odds ratios for 200000-300000 platelet count were $2.09(P=0.131)$ and $1.78(P=0.368)$, respectively. In addition, crude and adjusted (controlling for gender, DTB time, and type of MI) odds ratios for platelet count more than 300000 were $2.94(P=0.316)$ and $2.34(P=0.767)$, respectively.

At the end of the study, $161(73.85 \%)$ patients achieved

Table 1. Characteristics of Patients with Various Degrees of ST-Segment Depression

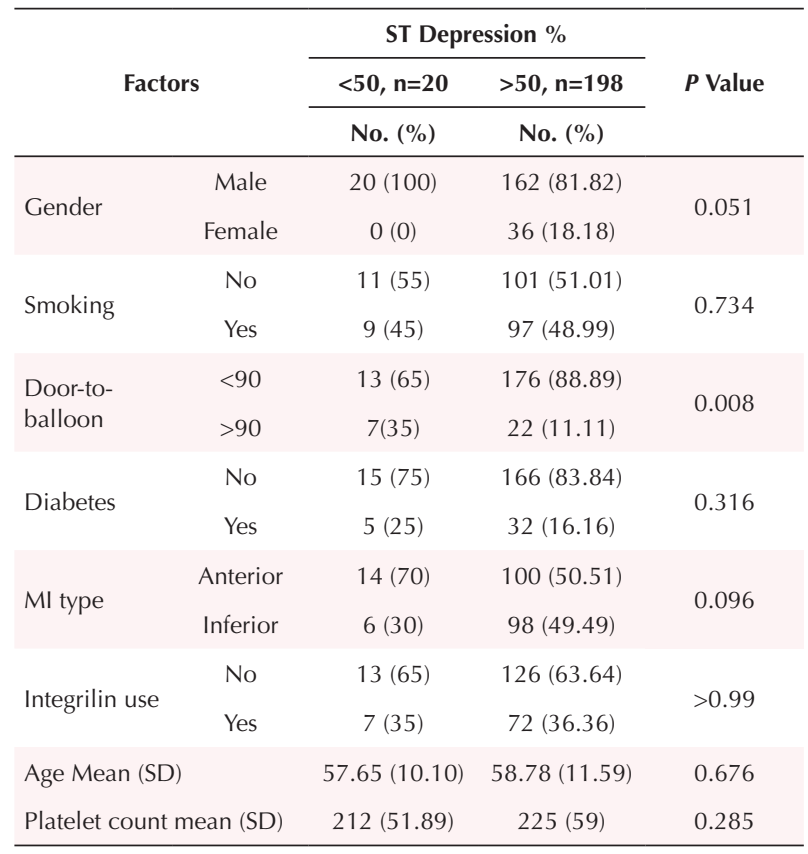

Table 2: Crude and Adjusted Associations Between Initial Platelet Count and the Degree of ST-Segment Depression

\begin{tabular}{|c|c|c|c|c|c|c|c|c|}
\hline \multirow{2}{*}{ Variable } & & \multicolumn{2}{|c|}{ SD Depression } & \multirow{2}{*}{$P$ Value } & \multirow{2}{*}{$\begin{array}{l}\text { Crude OR } \\
\text { (P Value) }\end{array}$} & \multirow{2}{*}{$95 \% \mathrm{Cl}$} & \multirow{2}{*}{$\begin{array}{c}\text { Adjusted OR } \\
\text { (P Value) }\end{array}$} & \multirow{2}{*}{$95 \% \mathrm{Cl}$} \\
\hline & & $<50$ & $>50$ & & & & & \\
\hline \multirow{3}{*}{$\begin{array}{l}\text { PLT count } \\
(* 1000 / \mu)\end{array}$} & $<200$ & $11(55)$ & $71(35.86)$ & \multirow{3}{*}{0.275} & \multicolumn{2}{|c|}{1} & 1 & \\
\hline & $200-300$ & $8(40)$ & $108(54)$ & & $\begin{array}{c}2.09 \\
(0.131)\end{array}$ & $0.80-5.45$ & $\begin{array}{c}1.78 \\
(0.368)\end{array}$ & $0.59-5.57$ \\
\hline & $>300$ & $1(5)$ & $19(9.60)$ & & $\begin{array}{c}2.94 \\
(0.316)\end{array}$ & $0.36-24.25$ & $\begin{array}{c}2.34 \\
(0.767)\end{array}$ & $0.27-113$ \\
\hline
\end{tabular}


Table 3. Characteristics of Patients with Various Degrees of Thrombolysis in Myocardial Infarction (TIMI)

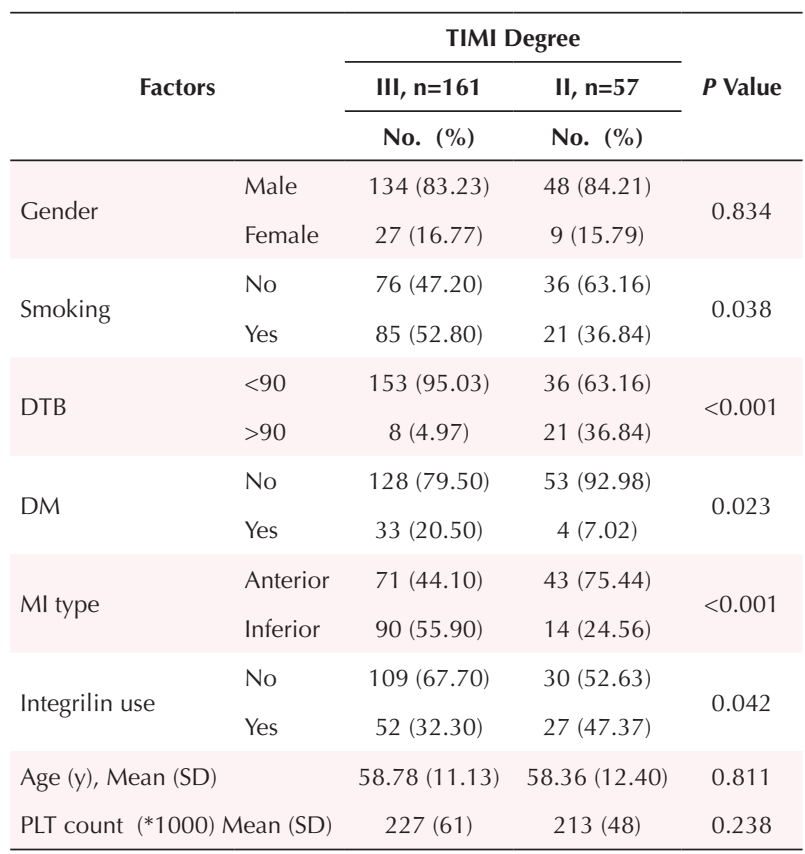

grade III thrombolysis, of whom 57 (26.15\%) subjects showed grade II thrombolysis. Frequency of cigarette smoking $(P=0.038)$, DM $\quad(P=0.023)$, and inferior type of MI $(P<0.001)$ among patients with grade III thrombolysis was higher than those in patients with grade II thrombolysis. On the other hand, patients with grade III thrombolysis had lower DTB time $(P<0.001)$ and Integrilin consumption $(P=0.042)$. Other factors were the same between the two groups (Table 3 ).

As can be seen in Table 4, there was no significant difference between patients with and without desired TIMI degree regarding platelet count $(P=0.380)$. The crude and adjusted (controlling for smoking, DTB time, $\mathrm{DM}$, Integrilin use, and type of $\mathrm{MI}$ ) odds ratios for platelet count of 200000-300000 were $0.76(P=0.394)$ and 0.70 $(P=0.354)$, respectively. The corresponding odds ratios for platelet count more than 300000 were $0.40(P=0.175)$ and $0.28(P=0.111)$, respectively.

\section{Discussion}

We observed no significant difference in platelet count of patients who underwent PCI with desired depression of the ST-segment in comparison with patients who did not show improvement in the ST-segment at the beginning of the treatment. Moreover, the platelet count was not different between patients with desired and low TIMI grades.

Ly et al showed that high platelet count during MI led to ST elevation and poor clinical outcomes such as death, recurrent MI, and congestive heart failure. ${ }^{11}$ The study carried out by Nikolsky et al demonstrated that the success rate of PCI was independent of the platelet count, but the incidence of thrombosis was constantly increased by platelet count elevation. They observed higher annual mortality rate $(P=0.02)$ or re-infarction $(P<0.001)$ among patients with platelet count more than 234000 compared to those with lower counts. ${ }^{12}$ Both of the mentioned studies had longer follow-up times, which could be a reason for difference in those results with the findings of the current study. It seems that platelet count is not associated with the short-term angiographic and electrocardiographic outcomes in patients with MI, but it can be a predictor for success rates in long term. However, similar to our findings, Sharif et al reported no difference of coronary blood flow and ST-segment depression between patients with different platelet counts; but those with platelet count more than 400000 had poorer myocardial perfusion and lower ejection fraction. The best myocardial perfusion was found in platelet count less than $200000 .{ }^{13}$ Finally, Kaplan et al showed significant relationship between high platelet count and low TIMI and high mortality rate, indicating the ability of the platelets in prediction of the angiographic outcomes of MI patients receiving PCI. ${ }^{14}$

The association between mean platelet volume and shortterm/long-term consequences of MI has been investigated by several studies that have reported controversial results. ${ }^{15,16}$ Other researchers have investigated the relationship between other platelet indices and severity of the coronary artery diseases. For example, Verdoia et al reported that immature platelet fraction was not associated with occurrence and severity of the coronary disorders. ${ }^{17}$ Platelets have critical role in coronary thrombosis and related diseases. ${ }^{18}$ Thus, different platelet indices have been assessed as predictors of coronary disorders, which revealed various results. A reason for such controversies is that these parameters are related with thrombopoiesis and other characteristics, such as age and DM, and do not act as independent risk factors.

The main limitation of the current study is its short follow-up time, which should be considered in future studies.

Table 4. Crude and Adjusted Associations Between Initial Platelet Count and the Degree of Thrombolysis in Myocardial Infarction (TIMI)

\begin{tabular}{|c|c|c|c|c|c|c|c|c|}
\hline \multirow{2}{*}{ Factors } & & \multicolumn{2}{|c|}{ TIMI Degree } & \multirow{2}{*}{$P$ Value } & \multirow{2}{*}{$\begin{array}{l}\text { Crude OR } \\
(P \text { Value })\end{array}$} & \multirow{2}{*}{$95 \% \mathrm{Cl}$} & \multirow{2}{*}{$\begin{array}{c}\text { Adjusted OR ( } P \\
\text { Value) }\end{array}$} & \multirow{2}{*}{$95 \% \mathrm{Cl}$} \\
\hline & & II & III & & & & & \\
\hline \multirow{3}{*}{$\begin{array}{l}\text { PLT count } \\
(* 1000)\end{array}$} & $<200$ & 25(43.86) & $57(35.40)$ & & 1 & & 1 & \\
\hline & $200-300$ & $29(50.88)$ & $87(54.04)$ & 0.380 & $\begin{array}{c}0.76 \\
(0.394)\end{array}$ & $0.40-1.43$ & $\begin{array}{c}0.70 \\
(0.354)\end{array}$ & $0.33-1.48$ \\
\hline & $>300$ & $3(5.26)$ & $17(10.56)$ & & $\begin{array}{c}0.40 \\
(0.175)\end{array}$ & $0.11-1.50$ & $\begin{array}{c}0.28 \\
(0.111)\end{array}$ & $0.06-1.34$ \\
\hline
\end{tabular}




\section{Conclusion}

Our results proved that platelet count cannot be considered as a predictor for electrocardiographic and coronary flow outcomes after PCI.

\section{Ethical Approval}

This study was approved by the Ethics Committee of Urmia University of Medical Sciences, Urmia, Iran (Code: ir.umsu.rec.1396.119).

\section{Conflict of Interest Disclosure}

None.

\section{Funding/Support}

None.

\section{Authors' Contributions}

Supervision, Investigation, Validation, Visualization, Data curation, Methodology, Resources, Software: RA, KK, T Y, AN MHSM, Statistical analysis: TY, MM, MA

Writing original draft, Writing review \& editing: $R A, K K, T Y$, AN, MHSM, MM, MK, MA.

All authors read and approved the final version of the manuscript for publication.

\section{Acknowledgments}

The authors are grateful to the all the participants for their sincere collaboration.

\section{References}

1. Zand Parsa AF, Ziai H, Haghighi L. The impact of cardiovascular risk factors on the site and extent of coronary artery disease. Cardiovasc J Afr. 2012;23(4):197-199. doi:10.5830/cvja-2011-052

2. Mohseni J, Kazemi T, Hosseinzadeh Maleki M, Beydokhti H. A systematic review on the prevalence of acute myocardial infarction in Iran. Heart Views. 2017;18(4):125-132. doi:10.4103/heartviews. heartviews_71_17

3. Yilmaz MB, Cihan G, Guray $Y$, et al. Role of mean platelet volume in triagging acute coronary syndromes. J Thromb Thrombolysis. 2008;26(1):49-54. doi:10.1007/s11239-007-0078-9

4. Fuster V, Badimon L, Badimon JJ, Chesebro JH. The pathogenesis of coronary artery disease and the acute coronary syndromes (1). N Engl J Med. 1992;326(4):242250. doi:10.1056/nejm199201233260406

5. Paul GK, Sen B, Bari MA, et al. Correlation of platelet count and acute ST-elevation in myocardial infarction. Mymensingh Med J. 2010;19(3):469-473.

6. Fox KA. Management of acute coronary syndromes: an update. Heart. 2004;90(6):698-706. doi:10.1136/ hrt.2003.020321

7. Hamm CW, Bassand JP, Agewall S, et al. ESC Guidelines for the management of acute coronary syndromes in patients presenting without persistent STsegment elevation: The Task Force for the management of acute coronary syndromes (ACS) in patients presenting without persistent ST-segment elevation of the European Society of Cardiology (ESC). Eur Heart
J. 2011;32(23):2999-3054. doi:10.1093/eurheartj/ ehr236

8. Wong CK, French JK, Gao W, White HD. Relation of initial platelet counts to thrombolysis in myocardial infarction-3 flow rates at 90 minutes after commencing fibrinolytic therapy in patients with acute myocardial infarction. Am J Cardiol. 2002;90(1):54-57. doi:10.1016/s0002-9149(02)02388-3

9. Turakhia MP, Murphy SA, Pinto TL, et al. Association of platelet count with residual thrombus in the myocardial infarct-related coronary artery among patients treated with fibrinolytic therapy for ST-segment elevation acute myocardial infarction. Am J Cardiol. 2004;94(11):14061410. doi:10.1016/j.amjcard.2004.08.015

10. Stone GW, Brodie BR, Griffin JJ, et al. Prospective, multicenter study of the safety and feasibility of primary stenting in acute myocardial infarction: in-hospital and 30-day results of the PAMI stent pilot trial. Primary Angioplasty in Myocardial Infarction Stent Pilot Trial Investigators. J Am Coll Cardiol. 1998;31(1):23-30. doi:10.1016/s0735-1097(97)00439-7

11. Ly HQ, Kirtane AJ, Murphy SA, et al. Association of platelet counts on presentation and clinical outcomes in ST-elevation myocardial infarction (from the TIMI Trials). Am J Cardiol. 2006;98(1):1-5. doi:10.1016/j. amjcard.2006.01.046

12. Nikolsky E, Grines CL, Cox DA, et al. Impact of baseline platelet count in patients undergoing primary percutaneous coronary intervention in acute myocardial infarction (from the CADILLAC trial). Am J Cardiol. 2007;99(8):1055-1061. doi:10.1016/j. amjcard.2006.11.066

13. Sharif D, Abu-Salem M, Sharif-Rasslan A, Rosenschein $U$. Platelet counts on admission affect coronary flow, myocardial perfusion and left ventricular systolic function after primary percutaneous coronary intervention. Eur Heart J Acute Cardiovasc Care. 2017;6(7):632-639. doi:10.1177/2048872616643690

14. Kaplan S, Kaplan ST, Kiris A, Gedikli O. Impact of initial platelet count on baseline angiographic finding and end-points in ST-elevation myocardial infarction referred for primary percutaneous coronary intervention. Int J Clin Exp Med. 2014;7(4):1064-1070.

15. Ghaffari S, Pourafkari L, Javadzadegan H, Masoumi N, Asghari Jafarabadi M, Nader ND. Mean platelet volume is a predictor of ST resolution following thrombolysis in acute ST elevation myocardial infarction. Thromb Res. 2015;136(1):101-106. doi:10.1016/j. thromres.2015.05.003

16. Sun XP, Li BY, Li J, Zhu WW, Hua Q. Impact of mean platelet volume on long-term mortality in Chinese patients with ST-elevation myocardial infarction. Sci Rep. 2016;6:21350. doi:10.1038/srep21350

17. Verdoia $M$, Nardin $M$, Rolla $R$, et al. Immature platelet fraction and the extent of coronary artery disease: a single centre study. Atherosclerosis. 2017;260:110115. doi:10.1016/j.atherosclerosis.2017.03.044

18. Larsen SB, Grove EL, Hvas AM, Kristensen SD. Platelet turnover in stable coronary artery disease - influence of thrombopoietin and low-grade inflammation. PLoS One. 2014;9(1):e85566. doi:10.1371/journal. pone.0085566 